\title{
Pattern Formation and Optimization in Army Ant Raids
}

\author{
Ricard V. Solé ${ }^{1,2}$, Eric Bonabeau ${ }^{2}$, \\ Jordi Delgado ${ }^{1,3}$, Pau Fernández ${ }^{1}$ and Jesus Marín ${ }^{3}$
}

\author{
(1) Complex Systems Research Group \\ Department of Physics, FEN \\ Universitat Politècnica de Catalunya \\ Campus Nord, Mòdul B4, 08034 Barcelona, Spain \\ (2) Santa Fe Institute, \\ 1399 Hyde Park Road, Santa Fe, NM87501, USA \\ (3) Departament de Llenguatges i Sistemes Informàtics \\ Universitat Politècnica de Catalunya, Campus Nord, Mòdul C6 \\ 08034 Barcelona (Spain)
}

Submitted to Proceedings R. Soc. London B

Keywords: Self-organisation, Army Ants, Pattern Formation, Optimization Running head : Army ant raid patterns 


\section{Summary}

Army ant colonies display complex foraging raid patterns involving thousands of individuals communicating through chemical trails. In this paper we explore, by means of a simple search algorithm, the properties of these trails in order to test the hypothesis that their structure reflects an optimized mechanism for exploring and exploiting food resources. The raid patterns of three army ant species, Eciton hamatum, Eciton burchelli and Eciton rapax, are analysed. The respective diets of these species involve large but rare, small but common, and a combination of large but rare and small but common, food sources. Using a model proposed by Deneubourg et al. (1989), we simulate the formation of raid patterns in response to different food distributions. Our results indicate that the empirically observed raid patterns maximise return on investment, that is, the amount of food brought back to the nest per unit of energy expended, for each of the diets. More-

over, the values of the parameters that characterise the three optimal pattern-generating mechanisms are strikingly similar. Therefore the same behavioural rules at the individual level can produce optimal colony-level patterns. The evolutionary implications of these findings are discussed.

\section{Introduction}

Swarm raid patterns in army ants are among the most astonishing social behaviours one can observe in nature. In a matter of hours, thousands of ants leave their bivouac, forming large columns or swarms, with the only purpose of finding food for the colony. These raids are able to sweep out an area of $1000 \mathrm{~m}^{2}$ in a single day. The "Huns and Tartars of the insect world" (Wheeler, cited in Hölldobler \& Wilson 1990, p. 573) are a beautiful example of decentralised control and self-organised behaviour (Bonabeau et al. 1997; see also Schneirla 1940). The term "Army Ant" (a.k.a. "legionary ants") refers essentially to the subfamilies Ecitoninae and Dorylinae, although behaviour patterns characteristic of those mentioned above are also found in the subfamilies Leptanillinae, Ponerinae and Myrmicinae (Hölldobler \& Wilson 1990). Such large-scale predatory patterns have been the subject of research by naturalists and myrmecologists since the past century: the structure of the patterns, the relation between division of labour and the raid patterns, their adaptive significance, etc. A sample of the reasons of the scientific interest in raid patterns can be found in (Hölldobler \& Wilson, 1990; chap. 16).

Different species of army ants display different raiding patterns. The column raiding 
of Eciton hamatum, the swarm raiding of E. burchelli and the intermediate pattern between columns and swarms- characteristic of the monomorphic species E. rapax would be good examples of the diversity in raid pattern structure (Burton \& Franks 1985). This diversity was explained by a theoretical model (detailed in section 2, see Deneubourg et al. 1989, 1990) which predicted that different patterns were the result of different food distributions and parameter combinations (according to prey preferences), though the underlying mechanism to build raid patterns was the same in all cases. These predictions were successfully tested by means of experiments with E. burchelli, whose characteristic swarming pattern changed, when prey distribution was modified, to look like the raiding pattern of E. rapax (Franks et al. 1991).

The mechanism behind raid patterns is a behavioural trait of army ants that has been subject to the forces of natural selection and, as such, it is "partially adaptive and partially an epiphenomenon created by stronger selection occuring on other traits" (Oster \& Wilson 1978; p. 314). The question we explore in this paper is about the "solution" found by the evolutionary process, that is, did selective forces optimize -in any welldefined sense- those patterns? Since we have a good, tested model of that mechanism, we will use it to look for answers to the above-mentioned question. In what sense are raid patterns optimal? We have chosen the quite reasonable measure of maximising the food input while minimising energy expenditure. This will be detailed in section 4, after a brief review of the search method in section 3. Finally, a discussion of the biological implications of our results will be provided in section 5 .

\section{Army ant raid model}

Following a previous work by Deneubourg et al. (1989), we consider a simple model of army ant raid patterns which involves individual, discrete units moving on a twodimensional discrete lattice where a pheromone field is created and maintained by the mobile units. More precisely, let $S_{k}(t)$ be the state of a given ant which will be either $S_{k}=1$ or $S_{k}=2$ if the ant is searching and moving away from the bivouac or returning back, respectively. Let $\phi(i, j) \geq 0$ be the trail concentration at lattice site $(\mathrm{i}, \mathrm{j})$. When ants are searching, they leave one unit of pheromone, unless the total amount of pheromone already exceeds a threshold value $\sigma_{1}$. When returning from exploration with a food item (returning ants always carry a food item) they leave $q$ units of pheromone; now the threshold is $\sigma_{2}$. The pheromone evaporates at a given decay rate $\delta$ : at each discrete time step $\phi(i, j) \rightarrow(1-\delta) \phi(i, j)$. Movement involves two different rules, following Deneubourg 


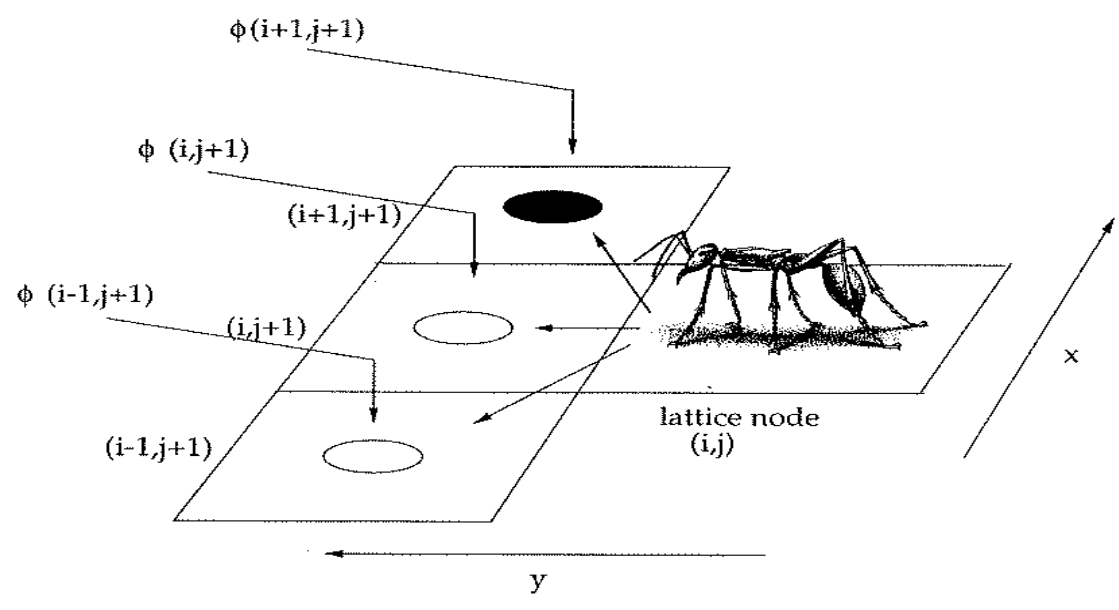

Figure 1: The movement of individual ants will depend on the amount of pheromone lying in the three neighboring sites in front of its current position (see text).

et al. (1989). These are:

1. Probability of movement. A given ant located at $(i, j)$ will move with a probability $P_{m}$ by depending on the pheromone field in the three grid points in front of $(i, j)$ (see figure 1) If the ant is leaving the nest, it reads:

$$
P_{m}=\frac{1}{2}\left[1+\tanh \left(\frac{\phi(i+1, j+1)+\phi(i+1, j)+\phi(i+1, j-1)}{\phi^{*}}-1\right)\right]
$$

(and similarly for returning ants but replacing $i+1$ by $i-1$ ). The parameter $\phi^{*}$ represents the concentration of trail pheromone for which the probability of moving per step is 0.5 . Here we take $\phi^{*}=100$ (changes in this parameter only shift the observed optimal solutions in parameter space but not the obtained patterns).

2. Once movement is allowed to occur, we have to chose one of the new grid nodes. The nodes with higher pheromone levels are more likely to be chosen. We have three probabilities $\pi_{L}, \pi_{0}$ and $\pi_{R}$ indicating left, central and right front nodes, respectively. These probabilities are:

$$
\begin{aligned}
& \pi_{L}=\frac{1}{C}[\mu+\phi(i+1, j+1)]^{2} \\
& \pi_{R}=\frac{1}{C}[\mu+\phi(i+1, j-1)]^{2}
\end{aligned}
$$


and obviously $\pi_{L}=1-\pi_{R}-\pi_{0}$. Here $C$ is given by

$$
C=[\mu+\phi(i+1, j+1)]^{2}+[\mu+\phi(i+1, j)]^{2}+[\mu+\phi(i+1, j-1)]^{2}
$$

This choice of a sigmoidal-like function is based on experiments (Franks et al. 1991). The parameter $\mu$ weights the attractiveness of empty nodes. If the chosen node is such that the total number of ants exceeds a maximum value $A_{m}$, then no movement is allowed to occur (here we use $A_{m}=30$ ). Two additional rules are required: (a) lost ants (i. e. those such that the reach the limits of the lattice) are removed from the system; (b) new ants enter from the bivouac, here located at $(1, L / 2)$. This is a fixed number (here we use $N_{b}=10$ ) but if the bivouac site is already at the limit $A_{m}$, no new ants are added. Other choices of the nonlinear (sigmoidal) function have been tested, for example

$$
\pi_{L}=\frac{1}{C}\left(\phi(i+1, j+1)^{2} /\left(\theta^{2}+\phi(i+1, j+1)^{2}+\phi(i+1, j)^{2}+\phi(i+1, j-1)^{2}\right)\right.
$$

where $\theta$ is a parameter and the rule 1 is transformed into a simple probabilistic decision of moving towards nearest nodes regardless the state of that node. These other choices gave basically the same results.

\section{Search algorithm}

In this section a simple search algorithm is introduced as a method of finding best-fit solutions. Most search approaches are based on genetic algorithms (Mitchell 1998) but here a simpler method is used (Marin and Solé 1999). In order to explore the 5-dimensional parameter space $\left(q, \mu, \delta, \sigma_{1}, \sigma_{2}\right)$ in search of possible optimal solutions, we need first to define a fitness measure which somehow takes into account that the maximum number of food items must be brought in at the smallest cost for the colony.

The search algorithm operates on a number of $G$ generations. Each generation consists of $N_{s}$ runs of the model, starting from a random set of parameter combinations. There is one parameter combination for each of the $N_{s}$ candidate "solutions", denoted by $i$. Solution $i$ is described by a 5 -vector $\left(q_{i}, \mu_{i}, \delta_{i}, \sigma_{1}(i), \sigma_{2}(i)\right)$. During a run of the simulation, which is performed on a $200 \times 200$ lattice, the following quantity is estimated over the $T=1000$ steps of the run:

$$
F_{i}=\sum_{t=1}^{T} \frac{\alpha_{\text {nest }}^{t}}{N_{\text {ants }}^{t}}
$$


i. e., at each time step t we measure the amount of food entering the nest $\left(\alpha_{\text {nest }}^{t}\right)$ divided by the total number of ants in the arena $\left(N_{\text {ants }}^{t}\right)$. This fitness measure introduces a reward proportional to the collected resources and a penalty proportional to the number of individuals involved in the search/recollection process.

We start by using $N_{s}=16$ candidate solutions that are (uniformly) generated at random from the following intervals: $1<q_{i}<50,0<\mu_{i}<50,10^{-3}<\delta_{i}<1,0<$ $\sigma_{1}<1000,0<\sigma<1000$. We run each simulation and compute the associated fitness $F_{i}\left(i=1, \ldots, N_{s}\right)$. Then a selection process is applied following the search method of Marin and Solé (1999):

1. Let $F^{*}=\frac{\sum_{j=1}^{N_{s}} F_{j}}{N_{s}}$. Solutions such that $F_{j}<F^{*}$ are removed from the system (these are called extinct solutions). If there are $N_{e}$ extinct solutions we should find $N_{e}$ new solutions to get again a complete population of $N_{s}$ solutions. This may be done in two different ways:

2. With probability $1-\tau$, a given extinct solution (say the $k$-th one) is replaced by one of the survivors (say the $j$-th one). All parameters are then modified by a small multiplicative amount $\epsilon$. If $\beta_{k}$ is the new value of one of the parameters of the new solution, it is updated as: $\beta_{k}=\beta_{j}(1 \pm \epsilon)$. If the new value is such that $\beta_{k}<\beta_{\text {min }}$ or $\beta_{k}>\beta_{\max }$ then we set the new value to $\beta_{k}=\beta_{\min }$ or $\beta_{k}=\beta_{\max }$, respectively. The survivor is chosen at random.

3. With probability $\tau$, a totally new random solution is generated.

The previous rules (here $\tau=0.2, \epsilon=0.2$ ) allow one to systematically explore the highdimensional parameter space. They have been shown to be very effective for a variety of fitness landscapes (Marín and Solé 1998).

\section{Results}

Three series of numerical experiments were performed using different combinations of food sources. Let $P_{f}$ the probability that a site be occupied by a food source of size $F$ (in arbitrary units). The following cases have been considered: (i) $P_{f}=0.01, F=200$ (e. g., wasp colonies, large but relatively rare); (ii) $P_{f}=0.5, F=1$ (e. g., scattered arthropods, common but small) and (iii) $P_{f}=0.25$ for $F=1$ and $P_{f}=1.25 \times 10^{-3}$ for $F=200$, (e. g., a combination of scattered arthropods and social insect colonies). All situations are 
simulated with the same total amount of resources. Here $N_{s}=16$ candidate solutions are evolved at each generation. In spite of the small number of solutions used, different simulations gave (for each $\left(F, P_{f}\right)$ ) the same average parameter values. For the first case,

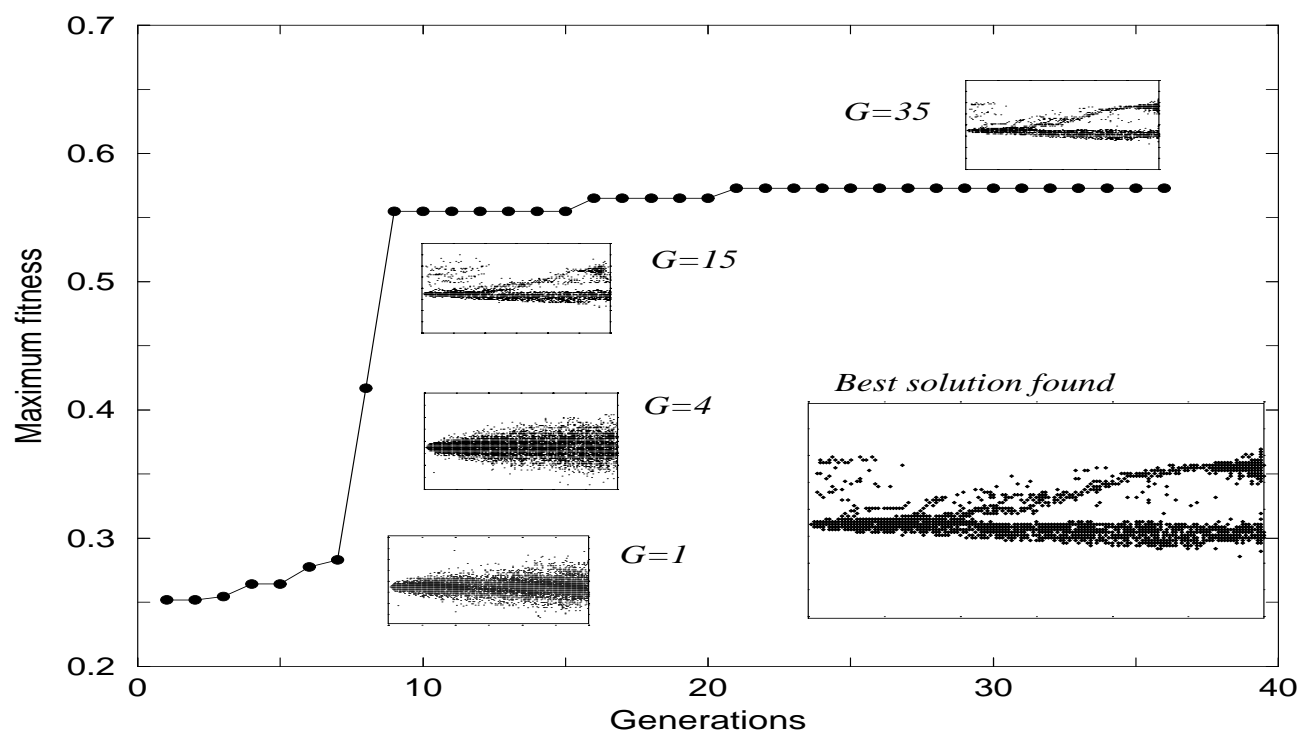

Figure 2: Solutions found in the case of large but rare food sources. This case would correspond to Eciton hamatum colonies. Note the random-walk shape found for small $G$ (first generations). The best solution found is consistent with E. hamatum raid patterns. See Table 1 for parameter values.

which would correspond to Eciton hamatum colonies, the search algorithm typically starts finding poor-fit solutions with random walk behaviour for individual ants (as we can see in figure 2.). In fact the envelope of the raid pattern $(G=1-4)$ as shown in the $(x, y)$-plane, is close to $y=\sqrt{x}$, as expected for a set of random walks. Later on $(G=15)$ we can see that the algorithm finds a solution displaying some short trails and a few generations later a multiple-trail pattern with a small amount of noise (as indicated by the ants scattered around large trails). This pattern is consistent with those characteristics of army ants feeding on large but rare food sources: only a few trails are observed.

When another, very different, situation is considered we find a rather different optimal search pattern, as it is shown in figure 3. Now the algorithm (after some random-like patterns, see $G=1-4$ ) finds branched solutions with many dendrites. Again, this corresponds to the foraging trails found in army ant species like Eciton burchelli. This dendritic pattern is in fact a fractal, and estimations of its fractal dimension give values of the fractal dimension $D_{f}$ in the range $1.5<D_{f}<1.7$. Finally, for the intermediate 


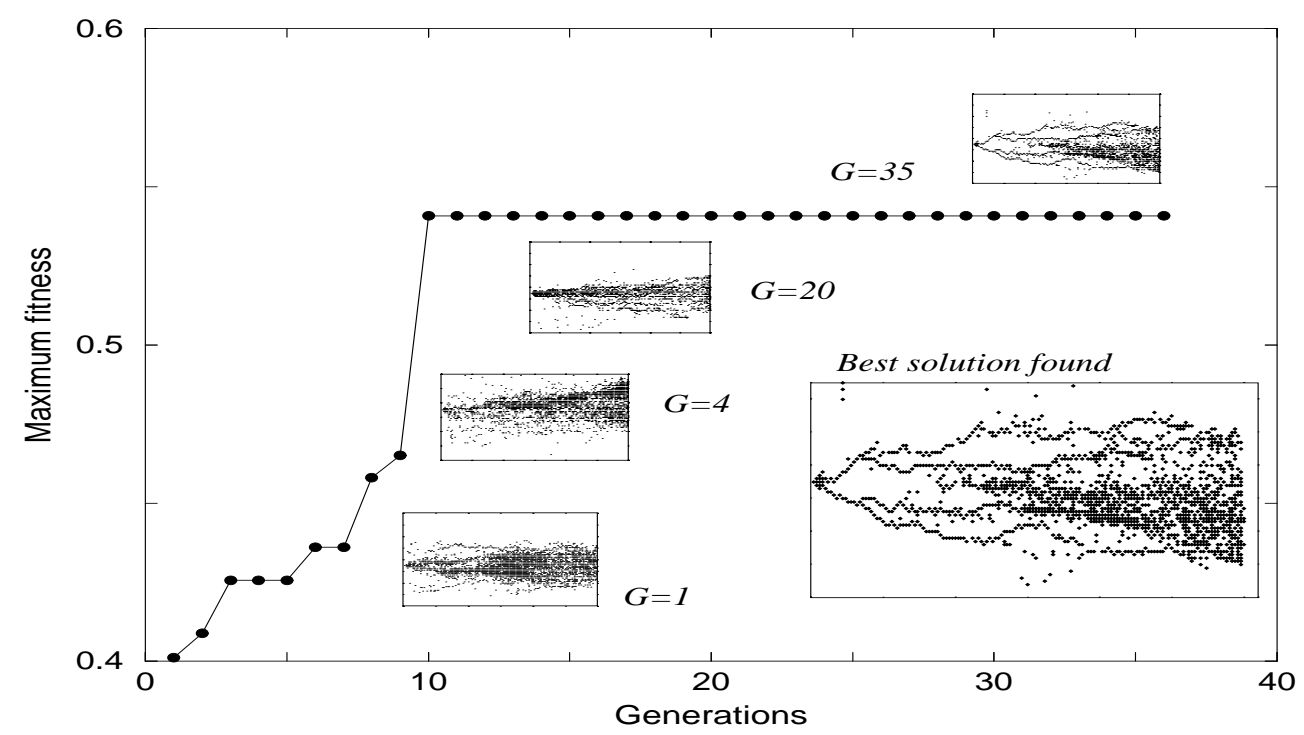

Figure 3: Solutions found in the case of small and frequent food sources, which would correspond to Eciton burchelli colonies. Again, the best solution found is consistent with E. burchelli raid patterns. See Table 1 for parameter values.

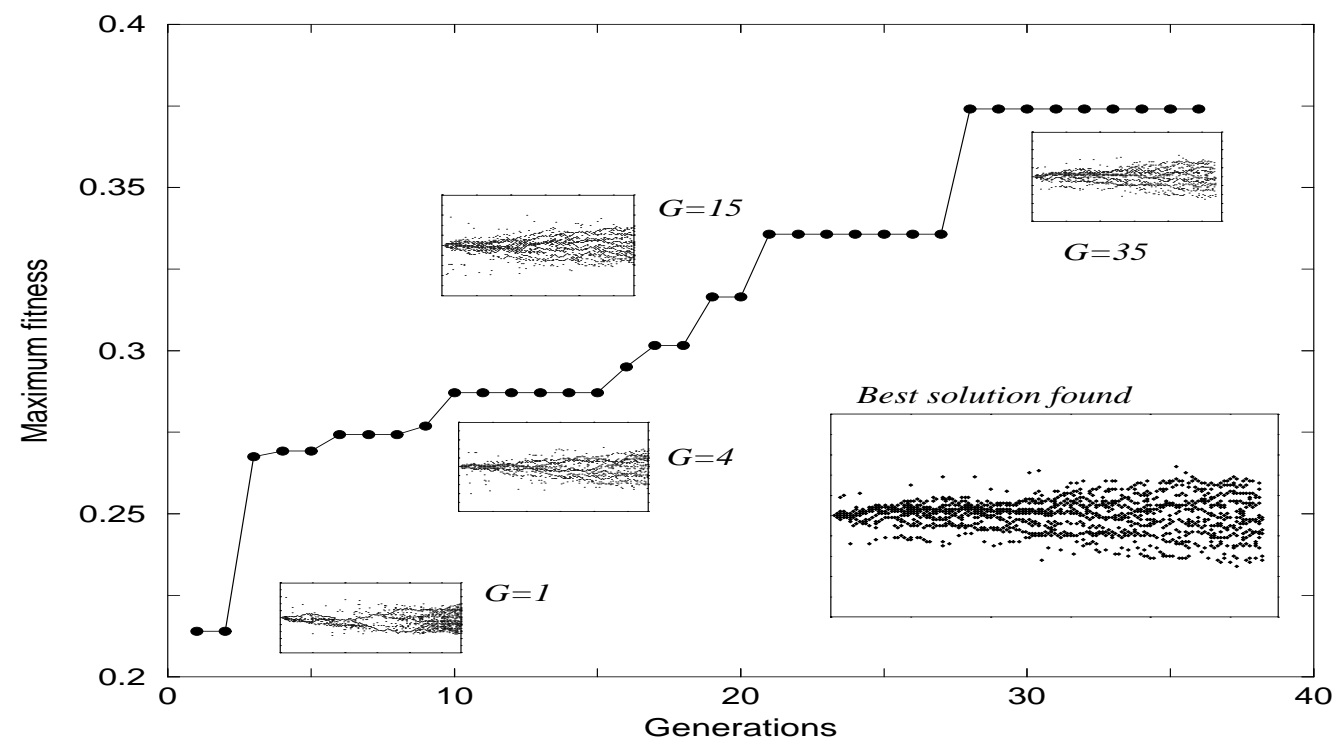

Figure 4: Solutions found for an intermediate configuration of food sources. Eciton rapax colonies are the natural analog of this case, with intermediate raid patterns between E. hamatum and E. burchelli. See Table 1 for parameter values. 
case we observe a mixed situation (figure 4): the system finds an optimal solution that is neither a single branch nor a set of well-separated dendrites. Clearly, an intermediate solution is found (although less fit than the previous ones), similar to the pattern observed in Eciton rapax. The parameter combinations obtained for the three different cases are

\begin{tabular}{|l|l|l|l|l|r|}
\hline Parameter & $\sigma_{1}, \sigma_{2}$ & $q$ & $\delta$ & $\mu$ & Spatial pattern \\
\hline Large, rare sources & 431,592 & 46 & $2.5 \times 10^{-3}$ & 47.5 & Single trails \\
\hline Poor, common sources & 51,390 & 47 & $2.1 \times 10^{-3}$ & 47 & Fractal \\
\hline Intermediate & 72,675 & 50 & $3.2 \times 10^{-3}$ & 48.5 & Few trails \\
\hline
\end{tabular}

Table 1: Results of the search algorithm. For each case, optimal parameters are indicated.

summarised in Table 1. We can see that in all cases the model finds good solutions that minimise pheromone decay, maximise pheromone laid but also the attractiveness of empty sites. In some sense, it seems that a good strategy need to both guarantee a persistent chemical trail and also to enable some flexibility to facilitate the exploration of new sites. We can also see that the main differences arises from the values of the pheromone saturation parameters: $\sigma_{1}$ and $\sigma_{2}$. In all cases, however, $\sigma_{1}<\sigma_{2}$.

\section{Discussion}

The results described in this paper are important for several reasons:

The patterns obtained through a plausible optimization procedure are strikingly similar to those observed in real colonies. That the simulated patterns have been obtained consistently in three different "ecological" conditions provides strong evidence that both the underlying model and the optimization procedure are appropriate.

The results show that different optimal patterns, in different conditions, can be obtained with similar values of the parameters, except for the two pheromone saturation parameters, $\sigma_{1}$ and $\sigma_{2}$. These two parameters may have been the two parameters tuned by evolution to make different species of army ants adapted to their respective ecological niches, represented here by spatial food distributions. This result is an important addition to Deneubourg et al. (1989) work: these authors showed that it is in principle possible to obtain the observed patterns with a single set of parameter values but they did not quantify the patterns in any way; while we were not able to quantify the spatial patterns per se, we did quantify their impact on foraging efficiency and did not rely solely on the eye of the beholder. The result of our more accurate quantification of the patterns is 
that different sets of parameter values are required to generate optimal patterns in different conditions, and we have identified which parameters values should be significantly different.

That $\sigma_{1}<\sigma_{2}$ is interesting because it translates into what is observed in army ants: army ant foragers lay pheromone while advancing with the swarm but to a lesser extent than when returning to the bivouac with prey. Although this inequality may not look like a surprising property since it makes sense to reinforce exploitation trails more than exploration trails, it is reassuring to find it as a natural consequence of maximising the foraging efficiency of the swarm.

Also, it makes sense to establish stronger trails to larger food sources, which is reflected in the large values of $\sigma_{1}$ and $\sigma_{2}$ obtained for large but rare food sources. For a given evaporation rate, lower rates of pheromone deposition lead to more flexibility, which may be useful when dealing with small, scattered food sources. A prediction of our study is that swarm raiders in Eciton hamatum should lay significantly more pheromone than in the other two species.

Assuming that the model reflects indeed what the ants do, which seems to be confirmed to a certain extent by a remarkable set of experiments (Franks et al. 1991), this suggests that self-organised raid patterns have been optimized in the course of evolution. Since raid patterns emerge out of relatively simple rules followed by individuals, it means that colonylevel selection has shaped the behaviour of individuals in such a way that individuals self-organise to implement optimal foraging strategies. Variation at the level of individual genotypes has been expressed in colony-level patterns through self-organisation.

\section{Acknowledgments}

This work has been supported by a grant DGYCIT PB97-0693 (RVS, JD), by a grant UPC PR98-11 (JD) and by the Santa Fe Institute. During this work, E. B. was the Interval Research Fellow at the Santa Fe Institute. 


\section{$6 \quad$ References}

1. Bonabeau E., Theraulaz G., Deneubourg J.-L., Aron S. \& Camazine S. 1997. Selforganization in social insects. Trends in Ecol. Evol. 12, 188-193.

2. Burton J.L. \& Franks N.R. 1985. The foraging ecology of the army ant Eciton rapax: An ergonomic enigma?. Ecol. Entom. 10, 131-141.

3. Deneubourg J.-L., Aron S., Goss S. \& Pasteels J.M. 1990. The self-organizing exploratory pattern of the Argentine ant. J. Insect Behav. 3, 159-168.

4. Deneubourg J.-L., Goss S., Franks N.R. \& Pasteels J.M. 1989. The blind leading the blind: Modeling chemically mediated army ant raid patterns. J. Insect Behav. $2,719-725$.

5. Franks N.R., Gomez N., Goss S. \& Deneubourg J.-L. 1991. The blind leading the blind in army ant raid patterns: Testing a model of self-organization (Hymenoptera: Formicidae). J. Insect Behav. 4, 583-607.

6. Hölldobler B. \& Wilson E.O. 1990. The Ants. Belknap Press of Harvard Univ. Press. Cambridge. Mass.

7. Marín J. \& Solé R.V. 1998. Macroevolutionary Algorithms: A New Optimization Method on Fitness Landscapes. IEEE Trans. Evol. Computation, in press.

8. Mitchell, M. 1998. An Introduction to Genetic Algorithms. MIT Press

9. Oster G.F. \& Wilson E.O. 1978. Caste and Ecology in the Social Insects. Princeton Univ. Press

10. Schneirla T.C. 1940. Further studies on the army-ant behavior pattern: Massorganization in the swarm-raiders. J. Comp. Psych. 25, 51-90.

11. Schneirla T.C. 1971. Raiding. In Topoff H.R. (ed.), Army Ants: A Study in Social Organization, W.H. Freeman, San Francisco. 